\title{
nejpec
}

\section{Economia e Criminalidade: uma Análise de Dados em Painel das Mesorregiões de Minas Gerais no Período 2005-2007}

\author{
Paula Andréa do Valle Hamberger \\ Professora de Economia \\ Universidade Federal de Goiás - UFG \\ E-mail: paulahamberger@gmail.com
}

\author{
Vanessa Marzano Araujo \\ Professora \\ Universidade Federal de Goiás Campus Catalão - UFG/Catalão \\ E-mail:
}

Ana Claudia Marques do Valle

Professora

Universidade Federal de Goiás - UFG

E-mail:

Resumo: Neste trabalho, investiga-se os efeitos de variáveis econômicas sobre as taxas de criminalidade em algumas mesorregiões selecionadas do Estado de Minas Gerais; de desenvolvendo uma análise comparativa entre as regiões de alta criminalidade, com os de baixas taxas de crimes violentos. Os modelos utilizados para analisar os dados consistem em dados em painel e foram rodados: Pooled, Efeitos Fixos e Efeitos Aleatórios. O estudo apontou que a faixa etária de 15 a 29 está mais propensa a cometer crimes.

Palavras-chave: Economia do Crime, Modelos de Dados em Painel

Abstract: In this work, we investigate the effects of economic variables on crime rates in selected mesoregions of the State of Minas Gerais; and we also develop a comparative analysis between regions of high crime, with those of low rates of violent crimes. The models used to analyze the data consist of panel data: Pooled, Fixed Effects and Random Effects. The paper pointed out that the age range of 15 to 29 is more likely to commit crimes.

Key words: Economics of Crime, Panel Data Models

JEL Code: K42, C23, C33 
p. 21 - Economia e Criminalidade: uma Análise de Dados em Painel das Mesorregiões de Minas Gerais no Período 2005-2007

\section{INTRODUÇÃO}

O problema apontado pelos indicadores de criminalidade vem cada vez mais atraindo a atenção tanto da população quanto do governo na tentativa de solucioná-lo. Porém, é recente a atenção dos pesquisadores ao tema. Isso pode explicar a escassez de estudos científicos que se dedicam a tentar explicá-lo e apontar direcionamentos que possam trazer soluções efetivas. O contexto em que se aplicam é evidentemente multidisciplinar envolvendo ciências como a sociologia, antropologia, psicologia, direito, economia, entre outras.

E, em confronto com a escassez de estudo temos a gravidade da questão que coloca o crime como um dos problemas centrais enfrentados pela sociedade. Nos estudos existentes a criminalidade tem sido associada com questões de gênero e de idade. Além desses fatores o aspecto econômico passou a assumir papel bastante relevante apresentando intima relação com a criminalidade. E, essa relação justificou e motivou o surgimento da Economia do Crime, área que se dedica a investigação empírica da criminalidade.

Os estudos econômicos sobre crime surgem enquanto discussão nos textos de Fleisher (1963, 1966), Smigel-Leibowitz (1965) e Ehrlich (1967), conforme Santos (2009). Mas foram os trabalhos de Becker (1968) e Erlich (1973) que levaram a investigação aos contornos de ciência, fundamentado a Economia do Crime.

Neste trabalho objetiva-se investigar os efeitos de variáveis econômicas sobre as taxas de criminalidade em algumas mesorregiões selecionadas do Estado de Minas Gerais; comparando os modelos explicativos para regiões de alta criminalidade, com os modelos de regiões com baixas taxas de crimes violentos.

\section{REFERENCIAL TEÓRICO}

De acordo com Beato (1998) os sociólogos definem as causas da criminalidade de duas maneiras divergentes. A primeira diz que criminalidade e violência se devem a fatores de natureza econômica; privação de oportunidades, desigualdade social e marginalização seriam estímulos decisivos para o comportamento criminoso. A outra credita ao delinquente e aos atos criminosos uma agressão ao consenso moral e normativo da sociedade; um baixo grau de integração moral produziria o fenômeno do crime.

Outros autores corroboram afirmando que quanto maior a desigualdade social, maior a violência, e observam que as cidades mais violentas do planeta têm como característica comum a desigualdade acentuada entre ricos e pobres. Fatores, como racismo, alcoolismo, drogas, facilidade de comprar armas e o baixo índice de escolaridade também pesam e agravam o problema.

O Projeto de Segurança Pública identifica como fatores que propiciam a prática da violência no Brasil, a pobreza relativa e moradia inadequada; o apoio familiar inconsistente; a deficiência de aprendizado; a exclusão da escola; a violência doméstica; as poucas oportunidades de emprego e exclusão econômica; a cultura da violência; a superlotação dos presídios; e, a inexistência de uma adequada política de drogas. 
No Brasil, a violência criminal atinge todos os segmentos sociais. Está presente entre os mais ricos até os mais pobres, o que faz da insegurança uma experiência compartilhada. Os crimes contra o patrimônio atingem, preferencialmente, os estratos sociais superiores e os crimes contra a pessoa vitimam em maior número os mais pobres, principalmente os jovens de 15 a 24 anos ou de 14 a 29 do sexo masculino e negro (Ministério da Justiça, 2011).

De acordo com o Projeto de Segurança Pública, nos anos de 2004 e 2005, das ocorrências registradas pelas Polícias Civis em todo o Brasil, verifica-se que os crimes violentos letais e intencionais e os não letais contra a pessoa passam por um período de estabilidade, os crimes violentos contra o patrimônio e delitos envolvendo drogas passam por um período de incremento e os delitos de trânsito passam por um período de redução significativa. Aproximadamente $42 \%$ dos crimes violentos letais e intencionais e $55 \%$ crimes violentos contra o patrimônio, ocorreram na região sudeste, enquanto $28 \%$ e $20 \%$, respectivamente, ocorreram na região nordeste. São Paulo foi o Estado onde ocorreu o maior número destes crimes e Roraima registrou o menor número de ocorrências (Ministério da Justiça, 2011).

Para reverter esta situação investimentos são realizados na segurança pública, entretanto não somente custos econômicos são gerados pela criminalidade. Observa-se que a criminalidade gera também custos sociais e políticos. Os efeitos sociais e político da criminalidade podem ser verificados na: erosão de capital social; transmissão de violência entre gerações; redução da qualidade de vida; comprometimento do processo democrático; redução na intensidade da relação entre as pessoas; redução na capacidade de formação de uma identidade de grupo entre os vizinhos; redução na vigilância informal dentro das comunidades; redução na sensação de segurança das pessoas em relação ao lugar onde residem; as pessoas limitam os locais onde transitam; deixam de ir a locais que gostam; evitam usar meios de transporte coletivo; evitam sair de casa à noite; gastam altas somas de recurso na proteção de suas residências; passam possuir armas e muitas vezes a andar armadas (Ministério da Justiça, 2011).

Pesquisa da Fundação Instituto de Administração (FIA), da Faculdade de Economia, Administração e Contabilidade da Universidade de São Paulo (USP), mostra que a maior parte dos investimentos em segurança são gastos em janelas e grades altas (48\%), em vigias (13\%) e em esquemas de fiscalização (9\%). Outro dado levantado é que cerca de $81 \%$ da segurança privada está nos bancos, órgãos públicos, indústrias e comércio (USP, 2006). Entretanto, segurança para todo tem que decorrer da ação eficiente do Estado e também da sociedade visando à redução das fontes que geram a violência e o crime.

Quanto ao Estado de Minas Gerais, foco desse estudo, diagnosticou-se que ao longo dos últimos anos, ocorreu uma significativa redução da taxa de crimes violentos praticados em Minas Gerais, conforme mostra o gráfico 1. Em termos percentuais, tal redução ficou em 17, 49\% entre 2005 e 2007. 
p. 23 - Economia e Criminalidade: uma Análise de Dados em Painel das Mesorregiões de Minas Gerais no Período 2005-2007

Gráfico 1: Taxa de Crimes Violentos por 100 mil habitantes em Minas Gerais

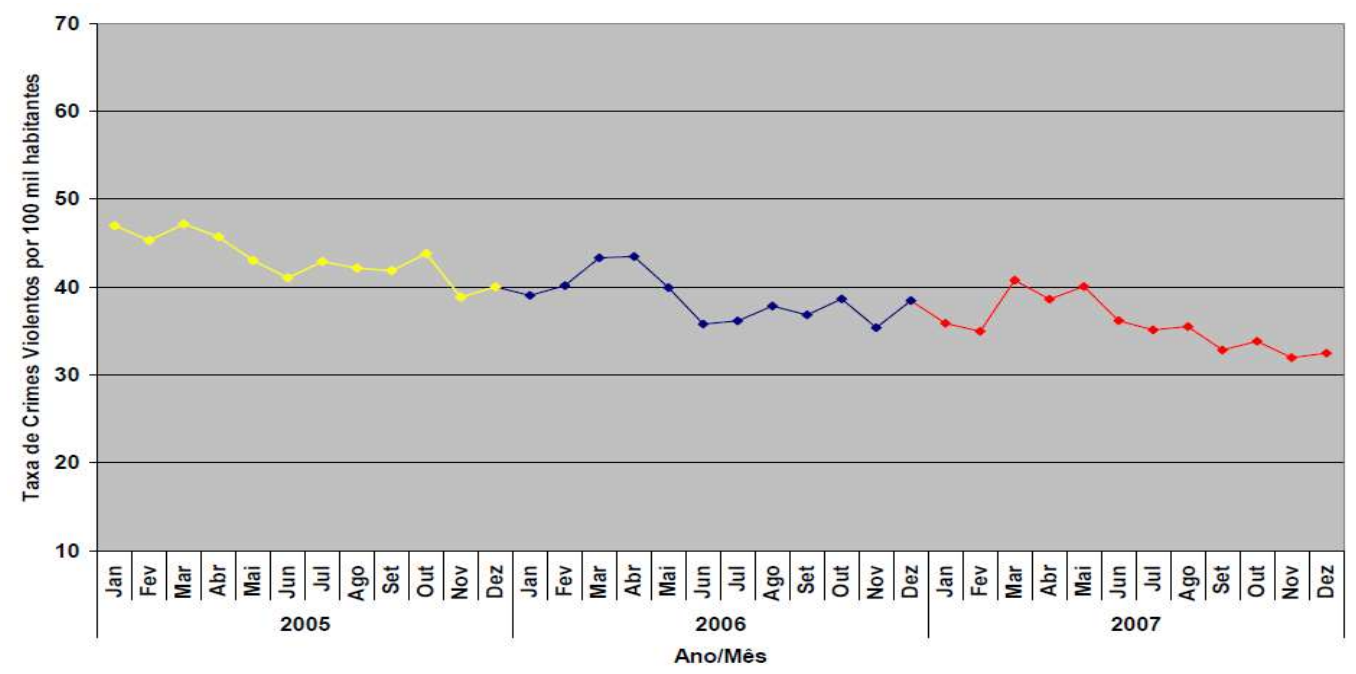

Fonte: Fundação João Pinheiro, 2011

Todavia, no que se refere aos termos espaciais houve consolidação da tendência já observada anterioremnte, ou seja, os municípios com maior concentração da criminalidade violenta são aqueles com maior número de habitantes. Nessa lista estão a Região Metropolitana de Belo Horizonte, a Região do Triângulo Mineiro e Alto Paranaíba, a Região Noroeste de Minas Gerais e também a Região Norte, principalmente no entorno de Montes Claros, conforme pode ser observado no Mapa 1.

Figura 1: Taxa Média de Crimes Violentos por 100 mil habitantes em MG durante o ano de 2007

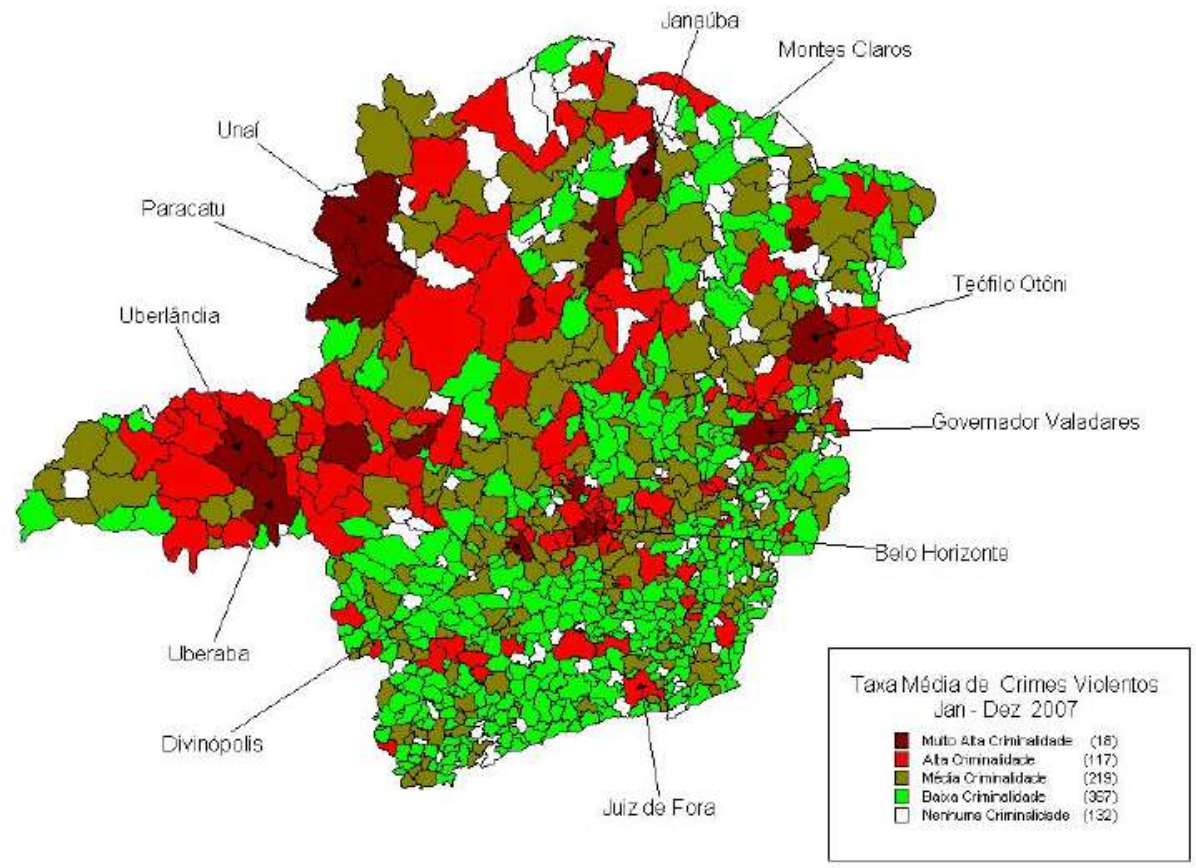

Fonte: Fundação João Pinheiro, 2011 


\section{METODOLOGIA}

Essa seção apresenta um estudo econométrico dos determinantes das taxas de criminalidade praticadas em Minas Gerais durante os anos de 2005 a 2007. Para tanto foram selecionadas 4 mesorregiões, entre elas estão aquelas que apresentaram os maiores e menores indicadores a fim de permitir comparações sobre seus determinantes. Os dados foram obtidos através do Índice Mineiro de Responsabilidade Social publicados pela Fundação João Pinheiro.

\subsection{Seleção das variáveis}

As variáveis selecionadas como possíveis determinantes básicos da taxa de criminalidade em Minas Gerais relacionadas as condições econômicas, sociais e demográficas das mesorregiões foram:

1 - Gasto per capita com atividades de educação (gasteduc) definido como valor dos gastos orçamentários apresentados nas prestações de contas anuais realizados no Ensino Fundamental, Ensino Médio, Ensino Superior, Ensino Profissional, Ensino Infantil e Educação de Jovens e Adultos dividido pela população;

2 - Rendimento médio do setor formal (rmsformal) definido como valor do rendimento médio dos empregados do setor formal no referido ano;

3 - Consumo per capita de energia elétrica (conseeletrica) definido como consumo anual per capita de energia elétrica na mesorregião durante o referido ano, medido em $\mathrm{KWH} / \mathrm{hab}$;

4 - Número de pessoas residentes com 15 a 29 anos de idade (popjovem);

5 - Densidadade populacional (denspop) definida como sendo a razão entre o número total de pessoas residentes e área do município;

6 - Gasto per capita com esporte e lazer (gastesporte)definido como sendo o valor dos gastos orçamentários apresentados nas prestações de contas anuais com atividades de esporte e lazer dividido pela população total do município nos referidos anos;

Portanto, a reta ajustada é a seguinte:

$Y=-$ gasteduc + rmsformal + conseeletrica + popjovem + denspop - gastesporte

A primeira variável tentou medir o efeitos nos gastos com atividade de educação. Espera-se que quanto maiores os gastos, melhor a qualidade do ensino e a possibilidade de aumento da renda e busca por um melhor emprego. O sinal esperado é quanto maior a taxa de criminalidade, menores são os gastos em educação.

A segunda variável foi inserida com motivação econômica e sua finalidade é mostrar que as atividades criminais dependem dos benefícios que possam gerar e devem superar os rendimentos obtidos no setor formal visto como custo de oportundade num montante que compense desrespeitar as leis. Portanto, espera-se uma correlação positiva. 
p. 25 - Economia e Criminalidade: uma Análise de Dados em Painel das Mesorregiões de Minas Gerais no Período 2005-2007

A terceira variável foi utilizada como proxy da taxa de crescimento econômico da mesorregião. Portanto, a previsão é de uma correlação positiva dos sinais, pois com o aumento do estoque de riqueza das vítimas pode-se esperar maior incidência dos crimes.

A quarta variável procurou mostrar o efeito da população jovem no modelo, uma vez que, numa perspectiva econômica, os jovens recebem menores salários, por isso teriam um custo de oportunidade menor ao exercer atividades criminosas. Além disso, há de se considerar que são menos propensos a controles sociais.

A quinta variável foi inserida com o intuito de mostrar que ocorre maior incidência de crimes em municípios onde a concentração populacional é maior. Portanto, espera-se uma correlação positiva, ou seja, quanto maior a desensidade populacional, maior a incidência de crimes.

A última variável procurou demonstrar que quanto maiores os gastos em atividades de esporte e lazer, menores são as incidências de crimes, mostrando uma correlação inversa. Sendo assim, a população teria outras atividades estimulantes e poderia preferir utilizar o tempo livre na prática de esportes e lazer ao invés de cometer crimes.

\subsection{Análise de dados via painel}

A notação matricial básica de análise de dados em painel é mostrada abaixo:

$$
\mathbf{y}_{i t}=\mathbf{x}_{i t}{ }^{\prime} \boldsymbol{\beta}+\mathbf{Z i}^{\prime} \boldsymbol{\alpha}+\varepsilon i t
$$

Em que:

yit - oferta de crimes com i

Os modelos utizados para analisar os dados em painel foram: Pooled, Efeitos Fixos e Efeitos Aleatórios. No modelo Pooled (empilhamento de dados) pressupõe-se que os regressores são exógenos. Sendo assim, se contém apenas um termo constante, o método dos Mínimos Quadrados Ordinários (MQO) é capaz de fornecer estimativas consistentes e eficientes de $\alpha$ e também aos demais parâmetros de $\beta$ associados às variáveis explicativas. Sendo assim, tanto o intercepto quanto as inclinações não variam. Porém, se zi é não observável e correlacionado com xit, a utilização de MQO deixa de ser eficiente e apresentará um resultado tendencioso e inconsistente devido a omissão da variável.

O modelo de Efeitos Fixos busca identificar um modelo com caracterisitcas especificas individuais, como:

$$
y_{\perp} \text { it }=\mathbf{x}_{\perp} \mathbf{i t}^{\mathbf{t}_{r}} \boldsymbol{\beta}_{\downarrow} \mathbf{t}+\left(\boldsymbol{\alpha}_{\mathrm{it}}+\varepsilon_{\mathrm{it}}\right)
$$

Para o modelo de Efeitos fixos (FE) o $\alpha_{i}$ e uma variavel aleatoria possivelmente correlacionada com xit. Portanto, o regressor $x_{i t}$ pode ser edogeno, em relacao ao $\alpha_{i}$, mas não ao $\varepsilon_{\text {it. }}$ Como por exemplo, educacao esta correlacionada com habilidade, que não varia com o tempo. 
Já o modelo de Efeitos Aleatorios, ou media da popupalcao, o $\alpha_{i}$ e puramente aleatorio (iid, $\left(0, \sigma^{2}{ }_{a}\right)$ ), sem correlacao com $x_{i t}$. Portanto, o regressor $x_{i t}$ e exogeno, sendo todos os estimadores de $\beta$ consistentes.

A literatura tambem indica a necessidade de estimar os modelos de pelo metodo de momentos generalizados desenvolvido por Arellano-Bond (1991) e Arellano-Bover (1995)/ Blundell-Bond (1998), estimadores que estao se tornando bastante populares. Amobos são estimadores gerais para apropriados para paines com pequeno $T$ e grande $\mathrm{N}$, ou seja, poucos periodos para um grande numero de individuos; com variaveis independentes que não são extritamente exogenas, ou seja, correlacionadas como o realizacoes passadas e presentes do erro; e, ainda com heteroscedasticidade, efeitos fixos e autocorrelacao entre os individuos.

\section{RESULTADOS E DISCUSSÕES}

Essa seção mostrará os resultados obtidos através da análise de dados em painel para as quatro mesorregiões selecionadas na análise.

\subsection{Região Metropolitana de Belo Horizonte}

Os resultados das regressões estimadas para a Região Metropolina de Belo Horizonte podem ser vistos na Tabela 1. Ao analisar o modelo foi possível observar que nem todas as variáveis selecionadas foram significativas para todos as variáveis depentes, por isso o método stepwise foi utilizado. A reta que melhor se ajustou ao modelo foi a primeira, em que a variável dependente constitui-se nos crimes violentos, pois apresentou o maior $R^{2}$. Sendo assim, ela foi selecionada para aplicação dos dados em painel.

A regressão que utiliza as taxas de crimes violentos não foram significativas as variáveis gastesporte e consumo de energia elétrica. A regressão seguinte com os dados de homicídio não apresentou significância para as mesmas variáveis do modelo anterior. Já o modelo que utiliza os dados dos crimes violentos contra as pessoas, além das variáveis anteriores, também não apresentou significância a variável popjovem. No modelo que utiliza os dados dos crimes violentos contra pessoa não foram signficativas as variáveis gasteduc e gastesporte. No modelo dos crimes violentos de menor potencial ofensivo apenas a variável rmsformal foi significativa.

A partir da seleção do modelo 1 e identificação de suas variáveis significativas, foi possível estimar as regressões utilizando-se os modelos pooled, efeitos fixos e efeitos aleatórios. 
p. 27 - Economia e Criminalidade: uma Análise de Dados em Painel das Mesorregiões de Minas Gerais no Período 2005-2007

Tabela 1 - Modelo testando todas as variáveis dependentes

\begin{tabular}{|c|c|c|c|c|c|}
\hline \multicolumn{6}{|c|}{$\begin{array}{l}\text { Região Metropolitana de Belo Horizonte } \\
\text { (t estatísticos estão representados abaixo dos coeficientes correspondentes) }\end{array}$} \\
\hline Variável dependente & crimviol & homicidio & cvpatrim & crcpessoa & cvmpo \\
\hline \multirow[t]{2}{*}{ constante } & -42.3599 & 6.56405 & -105.11 & 62.75122 & 356.0896 \\
\hline & -1.08 & 1.5 & -3.04 & 5.3 & 2.4 \\
\hline \multirow[t]{2}{*}{ gasteduc } & -0.15539 & -0.00969 & -0.13255 & -0.02284 & -0.08311 \\
\hline & -2.33 & -1.31 & -2.25 & -1.13 & 0.33 \\
\hline \multirow[t]{2}{*}{ Rmsformal } & 0.386597 & 0.021481 & 0.33631 & 0.050285 & 0.733013 \\
\hline & 10.43 & 5.2 & 10.26 & 4.49 & 5.23 \\
\hline \multirow[t]{2}{*}{ conseeletrica } & -0.08485 & -0.01499 & -0.00289 & -0.08196 & 0.164105 \\
\hline & -1.14 & -1.8 & -0.04 & -3.63 & 0.58 \\
\hline \multirow[t]{2}{*}{ popjovem } & -0.00068 & -0.00018 & -0.00045 & -0.00023 & 0.002573 \\
\hline & -1.84 & -4.46 & -1.38 & -3.63 & 1.84 \\
\hline \multirow[t]{2}{*}{ denspop } & 0.206886 & 0.019552 & 0.18063 & 0.026255 & -0.20765 \\
\hline & 6.65 & 5.64 & 6.56 & 2.79 & -1.76 \\
\hline \multirow[t]{2}{*}{ gastesporte } & -0.30046 & -0.05164 & -0.19022 & -0.1102 & -0.81757 \\
\hline & 0.72 & -1.1 & -0.51 & -0.87 & -0.51 \\
\hline Número observações & 315 & 315 & 315 & 315 & 315 \\
\hline R2 & 0,6738 & 0,2938 & 0,6864 & 0,2087 & 0,1164 \\
\hline
\end{tabular}

Tabela 2 - Estimativa dos Determinantes da Taxa de Crimes Violentos na Região Metropolitana de Belo Horizonte no período de 2005 a 2007

\begin{tabular}{|c|c|c|c|}
\hline \multicolumn{4}{|c|}{$\begin{array}{l}\text { Região Metropolitana de Belo Horizonte } \\
\text { (erros-padrão estão representados abaixo dos coeficientes correspondentes) }\end{array}$} \\
\hline \multirow[t]{2}{*}{ Variáveis } & \multicolumn{3}{|c|}{ Modelos } \\
\hline & Pooled & Ef. Fixos & Ef. Aleatórios \\
\hline \multirow[t]{2}{*}{ constante } & -69.69601 & -90.04787 & 76.04353 \\
\hline & 20.84038 & 24.24988 & 34.7283 \\
\hline \multirow[t]{2}{*}{ gasteduc } & -0.116396 & -0.161244 & -0.2162616 \\
\hline & 0.0428935 & 0.0140424 & 0.0754737 \\
\hline \multirow[t]{2}{*}{ rmsformal } & 0.3265883 & 0.3964836 & 0.1988124 \\
\hline & 0.0261198 & 0.026057 & 0.0482368 \\
\hline \multirow[t]{2}{*}{ popjovem } & 0.0010447 & -0.0008523 & -0.000163 \\
\hline & 0.0002518 & 0.0001524 & 0.0011196 \\
\hline \multirow[t]{2}{*}{ denspop } & 0.0826267 & 0.2209423 & 0.1861659 \\
\hline & 0.0210738 & 0.0296678 & 0.1126256 \\
\hline R2 & 0,6721 & 0,6823 & 0,0185 \\
\hline Obs & 315 & 315 & 315 \\
\hline Teste F (Chow) & & 165,38 & \\
\hline Teste Breusch e Pagan & & & 9,64 \\
\hline Teste de Hausman & & 20,61 & \\
\hline
\end{tabular}


Ao analisar os testes aplicados aos modelos de regressão para a Região Metropolitana de Belo Horizonte é possível observar que tanto o Teste de Chow quanto o Teste de Breusch Pagan indicam que existem efeitos não-observados afetando a taxa de crimes violentos, pois ambos rejeitam o Modelo Pooled. Sendo assim, é possível afirmar que as estimativas obtidas através desse modelo são tendenciosas e inconsistentes. Portanto, não devem ser levadas em consideração nas análises uma vez que o modelo omitiu variáveis que são importantes ao modelo e não foram observadas. Já o Teste de Hausman indica que o Modelo de Efeitos Fixos é melhor do que o Modelo de Efeitos Aleatórios.

\subsection{Regiao da Mesorregião do Triangulo Mineiro e Alto Paranaiba}

Os resultados das regressões estimadas para a Região do Triangulo Mineiro e Alto Paranaiba podem ser vistos na Tabela 3. Assim como ocorreu para a Regiao Metropolitcana de Belo Horizonte, ao analisar o modelo foi possível observar que nem todas as variáveis selecionadas foram significativas para todos as variáveis depentes, por isso o método stepwise foi utilizado, para selecionar o melhor modelo.

Tabela 3 - Modelo testando todas as variáveis dependentes.

(erros-padrão estão representados abaixo dos coeficientes correspondentes)

Regiao Mesorregiao do Triangulo Mineiro e Alto Paranaiba

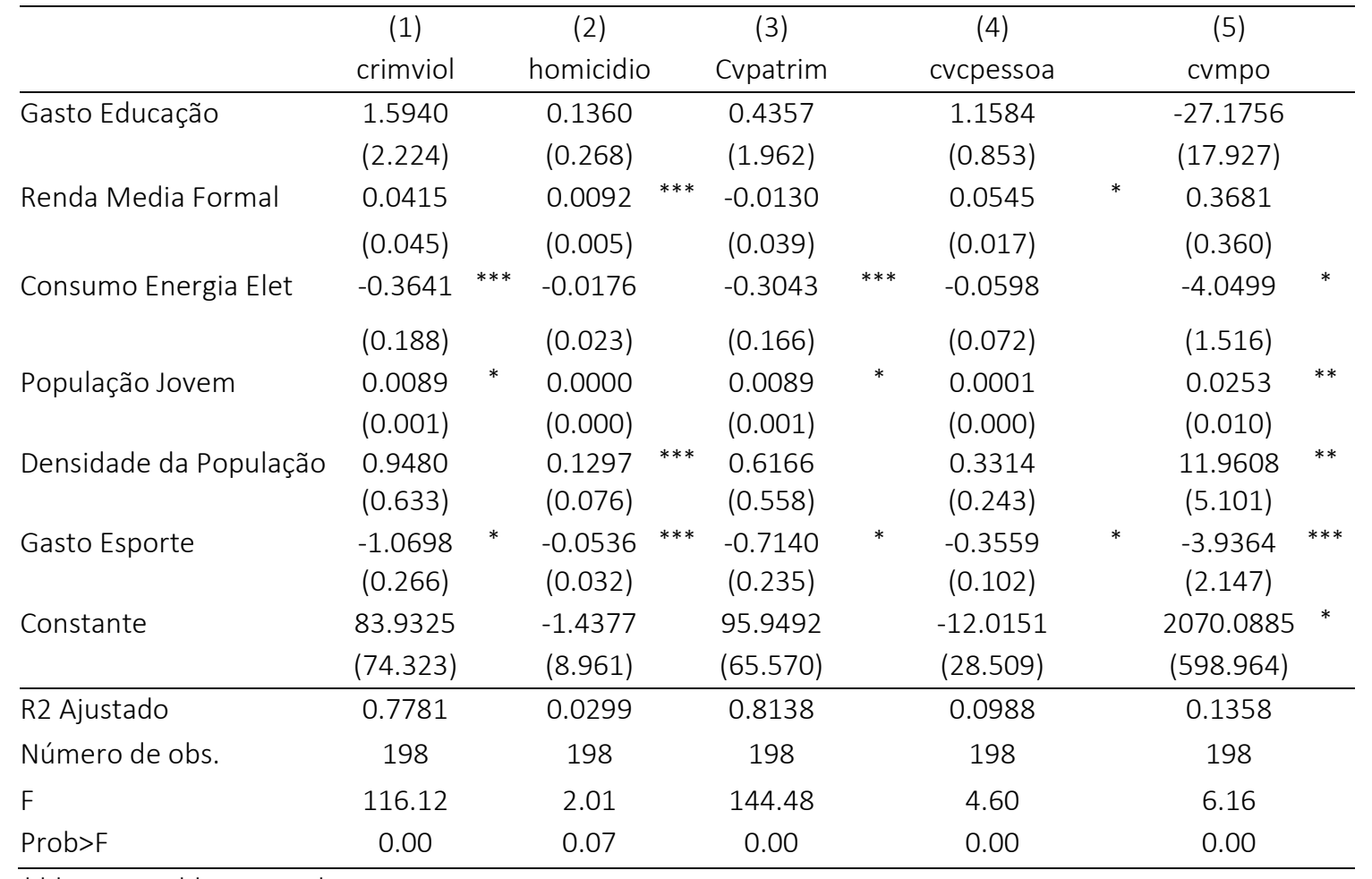

*** $\mathrm{p}<0.10, * * \mathrm{p}<0.05, * \mathrm{p}<0.01$

O modelo com crime violento (crimviol) como variavel dependente apresentou como sigfnificativas as variaveis gasto com esporte (gastesporte) e populcao jovem (popjovem), sendo estas tambem as variaveis significativas para crime contra 0 
p. 29 - Economia e Criminalidade: uma Análise de Dados em Painel das Mesorregiões de Minas Gerais no Período 2005-2007

patrimonio. Já os modelos com homicidio e crimes contra a pessoa apresentaram alem de gastesporte, tambem a variavel renda media formal (rmsformal) como A reta que melhor se ajustou ao modelo foi a que a variável dependente constitui-se nos crimes violentos contra o patrimonio, pois apresentou o maior $R^{2}$. Mas para efeitos de comparacao escolhemos o segundo maior $\mathrm{R}^{2}$, que refere-se a taxa de crimes violentos, em geral.

Ao analisar os testes aplicados aos modelos de regressão para a Região do Triangulo Mineiro e Alto Paranaiba é possível observar que tanto o Teste de Chow quanto o Teste de Breusch Pagan indicam que não existem efeitos não-observados afetando a taxa de crimes violentos, pois ambos aceitam o Modelo Pooled. Sendo assim, indica que não existem diferencas significativas entre os municipios, ou seja, não existem efeitos individuais significativos. Isso e confirmado pela estimativa de rho, que por ser muito baixa (.01828116) indica que as variacoes da criminalidade não podem ser explicadas por diferencas na criminalidade por municipio.

Tabela 4 - Estimativa dos Determinantes da Taxa de Crimes Violentos na Região Mesorregiao do Triangulo Mineiro e Alto Paranaiba no período de 2005 a 2007

Região Mesorregião do Triangulo Mineiro e Alto Paranaiba

(erros-padrão estão representados abaixo dos coeficientes correspondentes)

\begin{tabular}{|c|c|c|c|c|c|c|}
\hline & $\begin{array}{c}(1) \\
\text { Pooled }\end{array}$ & & $\begin{array}{c}(2) \\
\text { Efeitos fixos }\end{array}$ & & $\begin{array}{c}\text { (3) } \\
\text { Efeitos Aleatorios }\end{array}$ & \\
\hline Gasto Esporte & $\begin{array}{c}-0.8048 \\
(0.216)\end{array}$ & $*$ & $\begin{array}{c}-1.1615 \\
(0.041)\end{array}$ & $*$ & $\begin{array}{r}-0.6942 \\
(0.191)\end{array}$ & * \\
\hline População Jovem & $\begin{array}{l}0.0084 \\
(0.000)\end{array}$ & $*$ & $\begin{array}{l}0.0072 \\
(0.001)\end{array}$ & $*$ & $\begin{array}{l}0.0073 \\
(0.001)\end{array}$ & $*$ \\
\hline Constante & $\begin{array}{c}151.8116 \\
(8.263) \\
\end{array}$ & * & $\begin{array}{c}176.8324 \\
(4.938)\end{array}$ & $*$ & $\begin{array}{c}164.9361 \\
(12.993) \\
\end{array}$ & $*$ \\
\hline R2 Ajustado & 0.8266 & & 0.7778 & & & \\
\hline Número de obs. & 197 & & 198 & & 198 & \\
\hline $\mathrm{F}$ & 468.02 & & 403.45 & & & \\
\hline Prob $>F$ & 0.00 & & 0.00 & & 0.00 & \\
\hline Teste F (chow) & 1.20 & & & & & \\
\hline Teste Breusch e Pagan & 0.07 & & & & & \\
\hline Teste de Hausman & & & & & 2.27 & \\
\hline
\end{tabular}

(Para os modelos Ef. Fixos e Aleatórios é reportado R2 withingroups; $* * * p<0.10, * *$ $p<0.05, * p<0.01)$

Os dados revelam que os fatores explanatorios apresentam certa significancia estatisticas, com o gasto com esporte apresentando um efeito negativo sobre a criminalidade, ou seja, a medida que mais se despende recursos no desenvolvimento de atividades desportivas, cai a taxa de crimes violentos nos municipios da Regiao do Triangulo e Alto Paranaiba. E, a variavel populacao jovem apresenta um efeito positivo, indicando que uma populacao mais jovem leva a uma maior incidencia de crimes de maior violencia. 


\subsection{Região da Mesorregião Noroeste de Minas}

A Região Noroeste de Minas também é uma das regiões com os maiores índices de criminalidade. Por isso foi incluída no modelo. Os resultados das regressões estimadas para esse modelo podem ser vistos na Tabela 5.

Tabela 5 - Modelo testando todas as variáveis dependentes

\begin{tabular}{lccccc}
$\begin{array}{l}\text { Região Mesorregião Noroeste de Minas } \\
\text { (t estatísticos estão representados abaixo dos coeficientes } \\
\text { correspondentes) }\end{array}$ & & & \\
Variável dependente & crimviol & Homicídio & cvpatrim & cvcpessoa & cvmpo \\
& & & & & \\
\hline constante & 130.309 & 21.05061 & 54.77903 & 75.52149 & 1164.257 \\
& 1.29 & 1.61 & 0.77 & 1.39 & 2.04 \\
gasteduc & 0.024947 & 0.017461 & -0.05808 & 0.083039 & -0.90591 \\
& 0.17 & 0.93 & -0.57 & 1.07 & -1.11 \\
rmsformal & 0.069685 & -0.01918 & 0.058263 & 0.011431 & 0.373314 \\
& 0.48 & -1.02 & 0.57 & 0.15 & 0.45 \\
conseeletrica & -0.01888 & -0.00254 & -0.00658 & -0.0123 & -0.12749 \\
& -1.21 & -1.25 & -0.6 & -1.45 & -1.44 \\
popjovem & 0.015632 & 0.000754 & 0.012947 & 0.002685 & 0.068266 \\
& 6.79 & 2.52 & 7.99 & 2.16 & 5.24 \\
denspop & 0.015632 & 0.108793 & -1.93337 & -1.32138 & 36.55661 \\
& -0.47 & 0.12 & -0.4 & -0.35 & 0.93 \\
gastesporte & -0.82174 & 0.15931 & -0.42504 & -0.39678 & -3.62795 \\
& -1.05 & 1.57 & -0.77 & -0.94 & -0.82 \\
\hline Número observações & 57 & 57 & 57 & 57 & 57 \\
\hline R2 & 0.5461 & 0.2483 & 0.6625 & 0.1339 & 0.5179 \\
\hline
\end{tabular}

A Mesorregião Noroeste de Minas também não apresentou resultados significativos para todas as variáveis utilizadas no modelo. As três regressões que utilizaram as variáveis dependentes crimviol; em seguida cvpatrim e, por fim, cvmpo obtiveram como resultado apenas a variável popjovem como significativas. Já o modelo que utilizou como variável dependente homicidio apresentou significância para as variáveis gastesporte, popjovem e conseeletrica. Em seguida, o modelo com a variável dependente cvcpessoa apresentou como variávies significativas o conseeletrica e popjovem. A reta que apresentou melhor ajuste ao modelo foi cvpatrim. Todavia, foi escolhido o segundo maior R2 para dar sequência à análise de dados.

Em seguida, foram estimados os modelos pooled, efeitos fixos e efeitos aleatórios. Conforme resultados apresentados na Tabela 6. 
p. 31 - Economia e Criminalidade: uma Análise de Dados em Painel das Mesorregiões de Minas Gerais no Período 2005-2007

Tabela 6 - Estimativa dos Determinantes da Taxa de Crimes Violentos na Região Mesorregiao Noroeste de Minas no período de 2005 a 2007

\begin{tabular}{lrrr}
$\begin{array}{l}\text { Região Mesorregião Noroeste de Minas } \\
\text { (erros-padrão estão representados abaixo dos coeficientes }\end{array}$ \\
$\begin{array}{l}\text { correspondentes) } \\
\text { Variáveis }\end{array}$ & \multicolumn{1}{c}{$\begin{array}{l}\text { Modelos } \\
\text { Pooled }\end{array}$} & \multicolumn{1}{c}{ Ef. Fixos } & Ef. Aleatórios \\
\hline constante & 124.497 & 137.6165 & 138.246 \\
& 15.13722 & 15.23883 & 20.7958 \\
popjovem & 0.0147005 & 0.0141826 & 0.0140615 \\
& 0.0016986 & 0.0018261 & 0.002491 \\
\hline R2 & 0.6235 & 0.5323 & 0.0578 \\
Obs & 57 & 57 & 57 \\
Teste F (Chow) & & 60.32 & 5.43 \\
Teste Breusch e Pagan & & & 0.078 \\
Teste de Hausman & & & \\
\hline (Para os modelos Ef. Fixos e Aleatórios é reportado R2 within groups)
\end{tabular}

Ao analisar os testes aplicados aos modelos de regressão para a Região Noroeste de Minas é possível observar que tanto o Teste de Chow quanto o Teste de Breusch Pagan indicam que existem efeitos não-observados afetando a taxa de crimes violentos, pois ambos rejeitam o Modelo Pooled. Já o Teste de Hausman indica que o modelo de efeitos aleatórios é melhor do que o modelo de efeitos fixos.

\subsection{Regiao da Mesoregiao do Sul-Sudeste}

Diferentemente das duas outras regioes, a Mesorregiao do Sul-Sudeste apresenta uma das mais baixas taxas de crimes violentos, conforme pode-se observar no Mapa 1. E, foi escolhida para contrapor as analises anteriores, de forma a melhor fornecer um modelo que explique a baixa criminalidade.

Assim, como nas outras analises procedeu-se um abordagem stepwise com o objetivo de verificar quais as variaveis explicativas significativas para as diferentes variaveis dependentes: crime violento, homicidio, crime contra o patrimonio, crime contra a pessoa etc. O resultado da significancia destas variaveis se encontra apresentado na tabela 5. E, como pode-se observar varias variaveis apresentaram baixo poder estatistico para explicar a criminalidade. 
Tabela 7 - Modelo testando todas as variáveis dependentes.

(erros-padrão estão representados abaixo dos coeficientes correspondentes)

Regiao Mesorregiao do Sul-Sudeste.

\begin{tabular}{|c|c|c|c|c|c|c|c|c|c|c|}
\hline & $\begin{array}{c}(1) \\
\text { crimviol }\end{array}$ & & $\begin{array}{c}(2) \\
\text { homicidio }\end{array}$ & & $\begin{array}{c}\text { (3) } \\
\text { cvpatrim }\end{array}$ & & $\begin{array}{c}\text { (4) } \\
\text { cvcpessoa }\end{array}$ & & $\begin{array}{c}\text { (5) } \\
\text { cvmpo }\end{array}$ & \\
\hline Gasto Educação & $\begin{array}{l}0.1859 \\
(1.111)\end{array}$ & & $\begin{array}{l}0.0175 \\
(0.163)\end{array}$ & & $\begin{array}{l}0.2420 \\
(0.962)\end{array}$ & & $\begin{array}{c}-0.0562 \\
(0.378)\end{array}$ & & $\begin{array}{l}0.7997 \\
(9.401)\end{array}$ & \\
\hline Renda Media Formal & $\begin{array}{l}0.0225 \\
(0.015)\end{array}$ & & $\begin{array}{r}-0.0017 \\
(0.002)\end{array}$ & & $\begin{array}{l}0.0253 \\
(0.013)\end{array}$ & $* * *$ & $\begin{array}{c}-0.0028 \\
(0.005)\end{array}$ & & $\begin{array}{c}-0.0429 \\
(0.126)\end{array}$ & \\
\hline Consumo Energia Elet & $\begin{array}{c}-0.1421 \\
(0.131)\end{array}$ & & $\begin{array}{l}0.0041 \\
(0.019)\end{array}$ & & $\begin{array}{r}-0.1542 \\
(0.113)\end{array}$ & & $\begin{array}{l}0.0121 \\
(0.045)\end{array}$ & & $\begin{array}{c}-1.4959 \\
(1.109)\end{array}$ & \\
\hline População Jovem & $\begin{array}{l}0.0065 \\
(0.001)\end{array}$ & $*$ & $\begin{array}{l}0.0001 \\
(0.000)\end{array}$ & & $\begin{array}{l}0.0063 \\
(0.001)\end{array}$ & $*$ & $\begin{array}{l}0.0003 \\
(0.000)\end{array}$ & & $\begin{array}{l}0.0568 \\
(0.010)\end{array}$ & $*$ \\
\hline Densidade da População & $\begin{array}{l}0.1083 \\
(0.048)\end{array}$ & $* *$ & $\begin{array}{c}-0.0026 \\
(0.007)\end{array}$ & & $\begin{array}{l}0.0997 \\
(0.041)\end{array}$ & $* *$ & $\begin{array}{l}0.0086 \\
(0.016)\end{array}$ & & $\begin{array}{l}0.2930 \\
(0.403)\end{array}$ & \\
\hline Gasto Esporte & $\begin{array}{c}-0.1446 \\
(0.157)\end{array}$ & & $\begin{array}{l}0.0428 \\
(0.023)\end{array}$ & $* * *$ & $\begin{array}{c}-0.2148 \\
(0.136)\end{array}$ & & $\begin{array}{l}0.0702 \\
(0.054)\end{array}$ & & $\begin{array}{c}-0.6948 \\
(1.332)\end{array}$ & \\
\hline Constante & $\begin{array}{l}36.9268 \\
(32.240)\end{array}$ & & $\begin{array}{l}4.3489 \\
(4.739)\end{array}$ & & $\begin{array}{l}14.0263 \\
(27.903)\end{array}$ & & $\begin{array}{l}22.9019 \\
(10.966)\end{array}$ & $* *$ & $\begin{array}{l}759.8738 \\
(272.691)\end{array}$ & $*$ \\
\hline R2 Ajustado & 0.3157 & & 0.0023 & & 0.3569 & & 0.0043 & & 0.2504 & \\
\hline Número de obs. & 438 & & 438 & & 438 & & 438 & & 438 & \\
\hline $\mathrm{F}$ & 34.60 & & 1.16 & & 41.42 & & 1.32 & & 25.34 & \\
\hline Prob $>F$ & 0.00 & & 0.32 & & 0.00 & & 0.25 & & 0.00 & \\
\hline
\end{tabular}

$* * * p<0.10, * * p<0.05, * p<0.01$

O modelo escolhido que possui mais fatores explicativos foi a taxa de crimes violentos frente a regressores como a densidade populacional e a populacao jovem. Este modelo escolhido apresenta o maior $\mathrm{R}^{2}$ comparativo, contudo apresenta uma capacidade de explicacao da criminalidade muito baixa, de apenas $31 \%$.

Tabela 8 - Estimativa dos Determinantes da Taxa de Crimes Violentos na Região Mesorregiao do Sul-Sudeste no período de 2005 a 2007

Região Mesorregião do Triangulo Mineiro

(erros-padrão estão representados abaixo dos coeficientes

correspondentes)

\begin{tabular}{|c|c|c|c|c|c|c|}
\hline & $\begin{array}{c}(1) \\
\text { Pooled }\end{array}$ & & $\begin{array}{c}(2) \\
\text { Efeitos Fixos }\end{array}$ & & $\begin{array}{c}(3) \\
\text { Ef. Aleatórios }\end{array}$ & \\
\hline Densidade da População & $\begin{array}{l}0.0832 \\
(0.041)\end{array}$ & $* *$ & $\begin{array}{l}0.1120 \\
(0.051)\end{array}$ & & $\begin{array}{r}0.1156 \\
(0.062)\end{array}$ & $* * *$ \\
\hline População Jovem & $\begin{array}{l}0.0067 \\
(0.000)\end{array}$ & $*$ & $\begin{array}{l}0.0056 \\
(0.000)\end{array}$ & $*$ & $\begin{array}{l}0.0055 \\
(0.001)\end{array}$ & $*$ \\
\hline Constante & $\begin{array}{c}45.8627 \\
(3.087) \\
\end{array}$ & $*$ & $\begin{array}{c}55.8478 \\
(1.423) \\
\end{array}$ & $*$ & $\begin{array}{c}55.9282 \\
(5.000) \\
\end{array}$ & * \\
\hline R2 Ajustado & 0.4440 & & 0.3163 & & & \\
\hline Número de obs. & 438 & & 438 & & 438 & \\
\hline $\mathrm{F}$ & 175.52 & & 1503.93 & & & \\
\hline Prob $>F$ & 0.00 & & 0.00 & & 0.00 & \\
\hline Teste F (chow) & & & 4.10 & & & \\
\hline Teste Breusch e Pagan & & & & & 110.83 & \\
\hline Teste de Hausman & & & & & 3.08 & \\
\hline
\end{tabular}

$* * * p<0.10,{ }^{* *} p<0.05,{ }^{*} p<0.01$ 
p. 33 - Economia e Criminalidade: uma Análise de Dados em Painel das Mesorregiões de Minas Gerais no Período 2005-2007

Ao analisar os testes aplicados aos modelos de regressão para a Região do SulSudeste mineiro é possível observar que tanto o Teste de Chow quanto o Teste de Breusch Pagan indicam que existem efeitos não-observados afetando a taxa de crimes violentos, pois ambos rejeitam o Modelo Pooled. De forma que, indica que existem diferencas significativas entre os municipios, ou seja, existem efeitos individuais significativos. Isso e confirmado pela estimativa de rho, que apesar de não ser muito elevada apresenta valor não desprezivel (.51113799), ou seja, indica que as variacoes da criminalidade podem ser explicadas por diferencas na criminalidade por municipio.

\section{CONSIDERAÇÕES FINAIS}

Os resultados apontaram que o modelo economico do crime para as regioes selecionadas de Minas Gerais se ajusta melhor a populacao jovem, similarmente ao demonstrado nas conclusoes do trabalho de Oliveira (2003) aplicado para Brasilia. Ainda segundo este estudo, a populacao jovem, no estudo a faixa etaria de 15 a 29 anos, tem um impacto positivo sobre a criminalidade, pois este grupo esta mais propenso a cometer crimes; fato que se apresentou verdadeiro tanto para as regioes de maior criminalidade, como RMBH e Regiao do Triangulo Mineiro e Alto Paranaiba, quanto para as regioes de menor criminlaidade, como as Regioes Sul-Sudeste e Regiao Noroeste de Minas. Outro resultado interessante foi o impacto positivo do gasto com esporte sobre a criminalidade, o que sinaliza para as politicas publicas a importancia destes gastos.

Os resultados encontrados não foram satisfatorios, indicando que seria necessario uma nova etapa de alteracao das equacoes a serem estimadas; fato evidenciado pelo numero de variaveis que não apresentaram significancia estatistica, indicando que outra construcao da equacao poderia ser mais relevante. Assim, a proxima etapa seria utilizar as variaveis na forma logaritmica, com uma equacao no modelo loglog, de forma a padronizar as variaveis e minimizar os problemas de heteroscedasticidade. Outra etapa seria a utilizacao de modelos de momentos generalizados como o objetivo de avaliar o impacto de realizacoes passadas das variaveis explicativas e, da varivael dependente sobre a criminalidade corrente.

\section{BIBLIOGRAFIA}

Becker, G. S. (1968). Crime and punishment: An economic approach. The Journal of Political Economy, 76(2):169(217).

BEATO F., Claudio C. Determinantes da criminalidade em Minas Gerais. Rev. bras. Ci. Soc., São Paulo, v.13, n.37, $1998 . \quad$ Disponível em: <http://www.scielo.br/scielo.php?script=sci_arttext\&pid=S0102-4\&Ing=en\&nrm=iso>. Acesso em: 09 Jul 2011.

Ehrlich, I. (1967). The supply of illegimate activities. Unpublished manuscript, New York: Columbia University.

Ehrlich, I. (1973). Participation in illegitimate activities: A theoretical and empirical investigation. Journal of Political Economy, 81(3):526(536). 
Fajnzylber, P. \& Araujo Junior, A. (2001). Viol^encia e criminalidade. In Lisboa, M. B. \& Menezes Filho, N. A., editors, Microeconomia e Sociedade no Brasil, pages 333(394).

FUNDAÇÃO JOÃO PINHEIRO. Índice Mineiro de Responsabilidade Social. Disponível em $<w w w . f j p . g o v \cdot b r>$. Acesso em: 13 Jun 2011.

MINISTERIO DA JUSTIÇA. Implantação do Sistema Nacional de Estatísticas de Segurança Pública e Justiça Criminal. Disponível em: <http://www.mj.gov.br/senasp/estatisticas/mapacrime/Mapacrime2004_2005.pdf>. Acesso em: 05 Jul 2011.

OLIVEIRA, Andr_e L. Rossi de. Violence in The Capital of Brazil: An Analysis Based on the Economic Model of Crime. Working Paper no 295 Universidade de Brasilia, 2003.

MINISTERIO DA JUSTIÇA. Informações variadas. Disponível em: <http://www.mj.gov.br/senasp/estatisticas/custos/estat_custos_econ.htm>. Acesso em: 19 Jul 2011.

UNIVERSIDADE DE SÃO PAULO. Informações variadas. Disponível em: <http://www.usp.br/agen/repgs/2006/pags/096.htm>. Acesso em 15 Jul 2011. 